\title{
Neighboring Elemental Image Exemplar Based Inpainting for Computational Integral Imaging Reconstruction with Partial Occlusion
}

\author{
Bumseok Ko, Byung-Gook Lee, and Sukho Lee* \\ Department of Software Engineering, Division of Computer Information Engineering, Dongseo University, \\ Busan 617-716, Korea
}

(Received June 8, 2015 : revised June 29, 2015 : accepted July 16, 2015)

\begin{abstract}
We propose a partial occlusion removal method for computational integral imaging reconstruction (CIIR) based on the usage of the exemplar based inpainting technique. The proposed method is an improved version of the original linear inpainting based CIIR (LI-CIIR), which uses the inpainting technique to fill in the data missing region. The LI-CIIR shows good results for images which contain objects with smooth surfaces. However, if the object has a textured surface, the result of the LI-CIIR deteriorates, since the linear inpainting cannot recover the textured data in the data missing region well. In this work, we utilize the exemplar based inpainting to fill in the textured data in the data missing region. We call the proposed method the neighboring elemental image exemplar based inpainting (NEI-exemplar inpainting) method, since it uses sources from neighboring elemental images to fill in the data missing region. Furthermore, we also propose an automatic occluding region extraction method based on the use of the mutual constraint using depth estimation (MC-DE) and the level set based bimodal segmentation. Experimental results show the validity of the proposed system.
\end{abstract}

Keywords : Integral imaging, Image inpainting, Bimodal segmentation, Occlusion removal, 3-D visualization OCIS codes : (110.0110) Imaging systems; (110.6880) Three-dimensional image acquisition

\section{INTRODUCTION}

One of the advantages of the computational integral imaging (CII) is the capability of applying digital manipulation on the picked-up elemental images. Due to the digital manipulation, it is possible to reconstruct the 3-D image at a reconstruction plane of any desired depth. Furthermore, the recorded elemental images can be digitally post-processed.

This property enables the CII to reconstruct partially occluded 3-D objects for 3-D visualization and recognition [1-19]. Several occlusion removal methods have been studied in [15-19] to solve the problem of the degraded resolution of the computationally reconstructed 3-D image, which occurs due to the partial occlusion. In these studies, the occlusion region is detected by calculating the depth map. Then, the region corresponding to the depth map with relatively small depth values is regarded as the occluding region, and is removed by occlusion removal methods. However, even though the occluding object is removed, there remain data missing holes which prevent us from getting better visual qualities of the reconstructed images. Therefore, in [20], a linear inpainting based computational integral image reconstruction (LI-CIIR) method has been proposed, which fills in the data missing region caused by the occluding object by the linear inpainting technique [21] using the information of neighboring pixels.

It is shown experimentally in [20] that the LI-CIIR shows good results for images with objects having smooth surfaces. However, for the case that the object has a textured surface, the LI-CIIR cannot recover the textured data in the data missing region well. Furthermore, the LI-CIIR does not fully utilize the property that the same object region appears in several neighboring integral images.

Therefore, in this paper, we propose a neighboring elemental image exemplar based inpainting (NEI-exemplar inpainting) method which utilizes the original exemplar based inpainting method [22]. The exemplar based inpainting technique suits well the integral imaging application, since the source for the exemplar based inpainting can be found not only in the elemental image of interest but also in neighboring

\footnotetext{
*Corresponding author: petrasuk@gmail.com

Color versions of one or more of the figures in this paper are available online.
} 
elemental images. By using neighboring exemplars, the textures can be recovered in the data missing region.

In addition, we also propose an automatic occluding region extraction method which can automatically segment the occluding region based on the use of the mutual constraint using depth estimation (MC-DE) [23] and the level set based bimodal segmentation [24]. The MC-DE performs a stable calculation of the depth map based on the mutual constraint that exists between neighboring elemental images. The level set bimodal segmentation automatically segments the object and the background regions based on the competition between the depth values obtained by the MC-DE. Experimental results show the validity of the proposed system. The quality of the 3-D image reconstructed from the elemental image array with the occlusion removed by the proposed method is almost identical to that reconstructed from the elemental image array without occlusion.

\section{PROPOSED METHOD}

Figure 1 shows the overall diagram of the proposed system. First, the partially occluded 3-D object is picked up and recorded through a lenslet array to form the digital elemental image array. Then, the occluding region is segmented out by the mutual constraint using depth estimation (MC-DE) and the level set based bimodal segmentation. After that, we apply the proposed neighboring elemental image exemplar based inpainting (NEI-exemplar inpainting) to fill in the missing data inside the occluding region. Finally, the 3-D image is reconstructed using the CIIR. We explain the details of each step in the next sub-sections.

\subsection{Occluding Region Segmentation}

One of the major problems in occlusion artifact removal is the automatic extraction of the occluding region. Recently, several methods have been proposed for the extraction of the occluding region. One of the most successful methods is that which computes the depth map based on the mutual

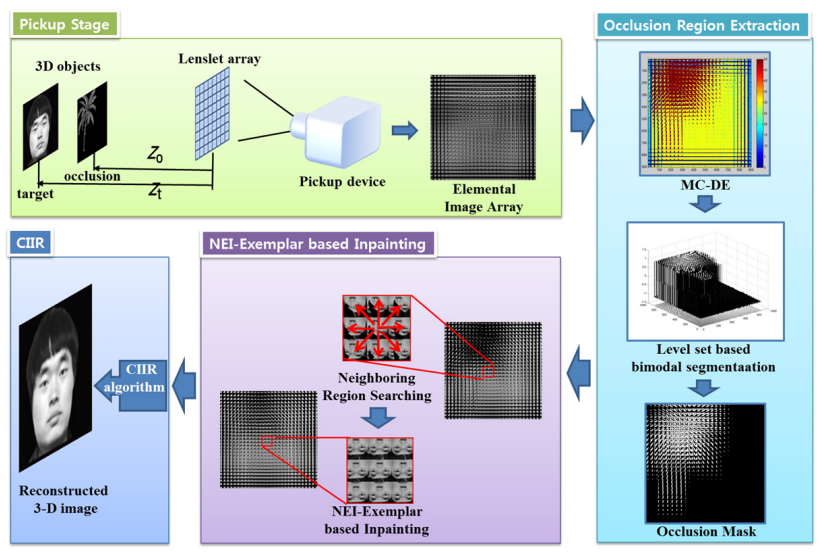

FIG. 1. Overall diagram of the proposed NEI-exemplar inpainting method. constraints between the elemental images [23]. Hereafter, we call this method the mutual constraint using depth estimation (MC-DE).

In the MC-DE method, it is assumed that the translation of the same object is constant between neighboring elemental images. Using the constraint between $(2 m+1) \times(2 m+1)$ neighboring elemental images, the problem of finding the disparity vector $(u, v)$ can be formulated as follows [23]:

$$
(\hat{u}(x, y), \hat{v}(x, y))=\arg \min _{u, v}\left(\sum_{n_{1}=-m}^{m} \sum_{n_{2}=-m}^{m}\left(I_{n_{1}, n_{2}}\left(x+n_{1} u, y+n_{2} v\right)-\bar{I}\right)\right)^{2},
$$

where $I_{n_{1}}, n_{2}$ denotes the $\left(n_{1}, n_{2}\right)$-th elemental image, $\bar{I}$ is the average all the intensity values $I_{n_{1}}{ }{ }_{n}$ for $n_{1}, n_{2}=-m \sim m$ and $(\hat{u}(x, y), \hat{v}(x, y))$ denotes the solution disparity vector at $(x, y)$, where $(x, y)$ is the position of the pixel under consideration. The disparity vector is computed for every pixel to form a disparity map.

Figure 2 (a) and (b) show the $\hat{u}$ and $\hat{v}$ components of the disparity vector obtained by applying (1) on the elemental image obtained by the optical settings shown in the pickup-stage of Fig. 1. The elemental image is composed of two objects ('tree' and 'face' objects) with different depths. It can be seen in Fig. 2 that the depth values of the 'tree' region and the 'face' region are well obtained by the MC-DE method.

Applying a thresholding process on the figures in Fig. 2 with a suitable threshold value can discriminate the occluding region from the object region. However, it is not clear which threshold value discriminates the two regions well. Therefore, in this paper, we propose to utilize the level set based bimodal segmentation method. This method has been proposed by us in [24] to automatically segment target regions without using a pre-defined threshold value. The bimodal segmentation performs a two-phase segmentation based on the competition between the brightness values in the image.

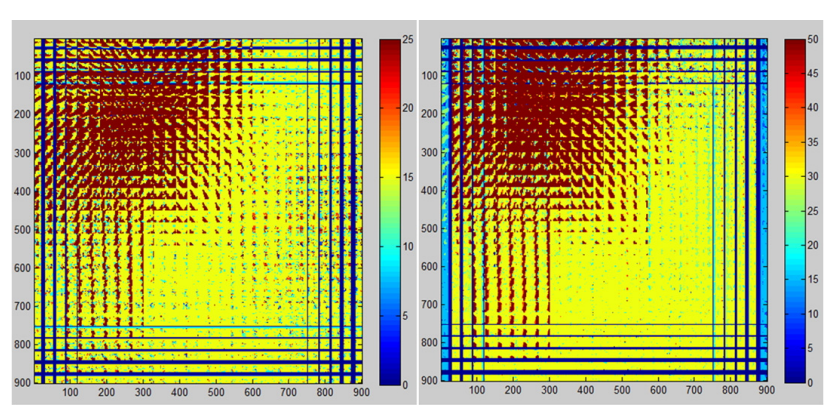

(a)

(b)

FIG. 2. Disparity vector map obtained by the MC-DE method (a) $\hat{u}$ component of the disparity vector (b) $\hat{v}$ component of the disparity vector. 
The level set based bimodal segmentation performs a two level segmentation by minimizing the following energy functional with respect to the level set function $\phi$ :

$$
\begin{aligned}
E(\boldsymbol{\phi}) & =\int_{\Omega}\left|u_{0}(\mathbf{r})-a v e_{\{\phi \geq 0\}}\right|^{2} \phi(\mathbf{r}) H(\alpha+\phi(\mathbf{r})) d \mathbf{r} \\
& -\int_{\Omega}\left|u_{0}(\mathbf{r})-a v e_{\{\phi<0\}}\right|^{2} \phi(\mathbf{r}) H(\alpha-\phi(\mathbf{r})) d \mathbf{r}
\end{aligned}
$$

Here, $u_{0}$ denotes the brightness value of the pixel, $\mathbf{r}$ denotes the position vector of the pixel, ave $\{\phi \geq 0\}$ and $a v e\{\phi<0\}$ denote the average brightness values in the regions in $u_{0}$ corresponding to the regions $\{\mathbf{r} \mid \phi(\mathbf{r}) \geq \mathbf{0}\}$ and $\{\mathbf{r} \mid \phi(\mathbf{r})<\mathbf{0}\}$, respectively, and $\alpha$ is an arbitrary positive value. $H(\cdot)$ is the Heaviside step function defined as follows:

$$
H(a)=\left\{\begin{array}{l}
1 \quad \text { if } a \geq 0 \\
0 \quad \text { if } a<0
\end{array}\right.
$$

For the problem of segmenting the object region based on the depth map, we set $u_{0}$ as the depth map instead of a normal image. That is, we let

$$
u_{0}(\mathbf{r})=\sqrt{\hat{u}(\mathbf{r})^{2}+\hat{v}(\mathbf{r})^{2}}
$$

where $\hat{u}(\mathbf{r})$ and $\hat{v}(\mathbf{r})$ are the components of the disparity vector calculated by $(1)$, and $\mathbf{r}=(x, y)$.

The level set value at the $\mathbf{r}$, i.e., $\phi(\mathbf{r})$ converges to $\boldsymbol{\alpha}$ or $-\alpha$ depending on the value of $u_{0}$.

Figure 3 shows the application of the level set bimodal segmentation on the depth map $u_{0}$ where we set $\boldsymbol{\alpha}=1$.
It can be seen in Fig. 3 that the level set function automatically converges to the state of 1 or -1 , since $\alpha=$ 1. That is, the level set function value converges to a positive or negative value depending on whether the pixel $\mathbf{r}=(x, y)$ belongs to the occluding or the target region.

Thus, collecting the pixels with positive values of the level set function, we can make a weight mask image which indicates the occluding region. The resulting weight mask image is shown in Fig. 3 (h).

\subsection{Data Reconstruction with Neighboring Elemental Image Exemplar based Inpainting}

Image inpainting is a technique which fills in missing values with reliable data obtained from the boundary of the missing region. In the smoothing based inpainting technique the data from the boundary is smoothed into the missing region to fill in the inpainting region. In [20], we proposed an occluding region removal method based on this inpainting technique to fill in the missing data in the occluding region.

However, if the original data contains many textural data, they cannot be recovered with the smoothing based inpainting. To fill in the inpainting region with textured data, an exemplar based inpainting technique has been proposed for normal images in [22].

Normally, the textured region in the background appears better with exemplar based inpainting than with the smoothing based inpainting. However, there is no guarantee that the textured regions recovered by the original exemplar based inpainting technique are the original data, because no one knows what was behind the occluded region. In comparison, in the case of integral imaging, there exists the additional advantage that the same data appears across several neighboring elemental images.

In this work, we modify the original exemplar based

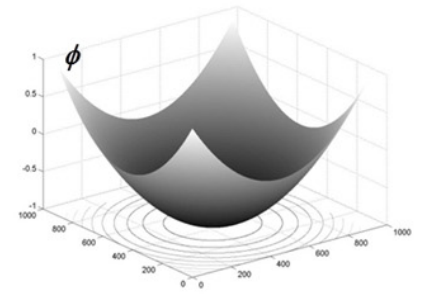

(a)

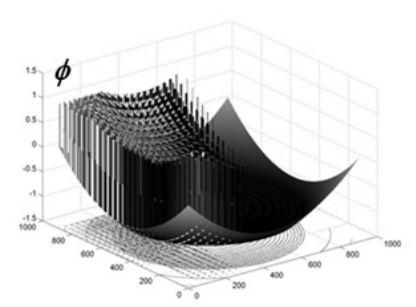

(e)

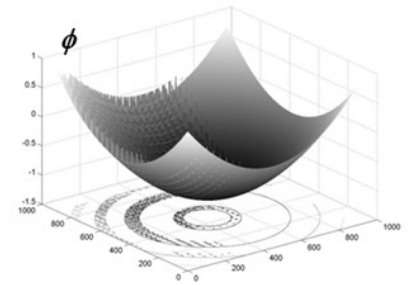

(b)

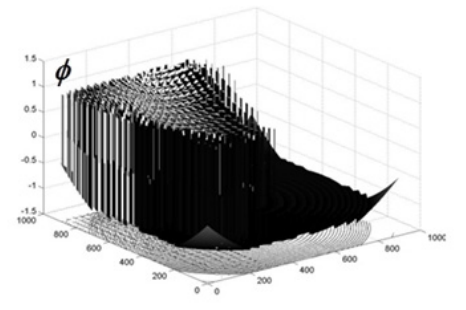

(f)

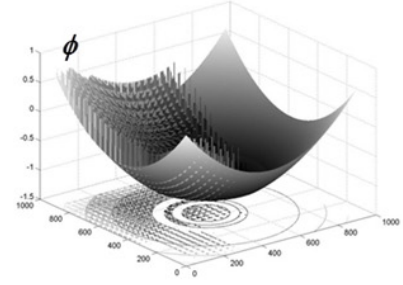

(c)

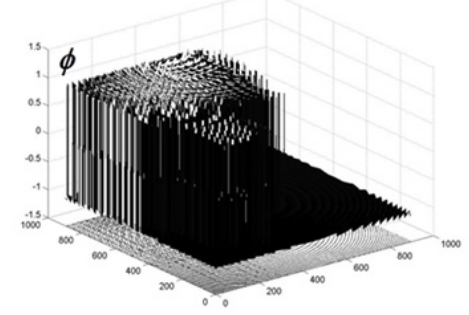

(g)

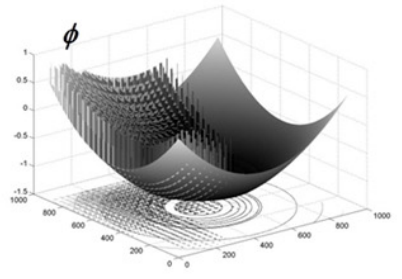

(d)

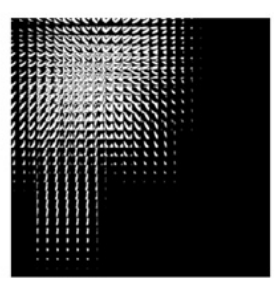

(h)

FIG. 3. Obtaining the weight mask by the level set based bimodal segmentation method (a) (g) evolution of the level set function $(\phi)(\mathrm{h})$ resultant weight mask. 


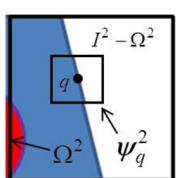

(a)

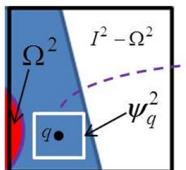

(e)

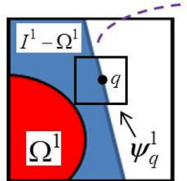

(b)

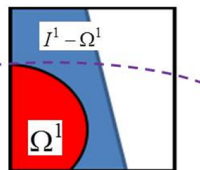

(f)

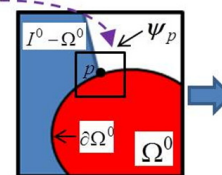

(c)

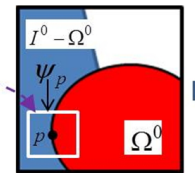

(g)

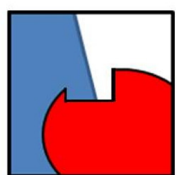

(d)

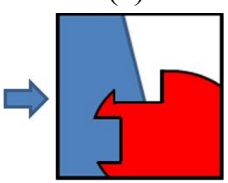

(h)
FIG. 4. Explaining the exemplar based inpainting process of the proposed method. (a)(e) second left neighboring elemental image (b)(f) first left neighboring elemental image (c) $(g)$ elemental image under consideration $(d)(h)$ inpainted result. Top row: first inpainting step Bottom row: second inpainting step.

inpainting technique to recover the textured regions in the occluding region using the above-mentioned advantage. We call the modified exemplar based inpainting technique the neighboring elemental image exemplar based inpainting (NEI-exemplar inpainting). Figure 4 shows the concept of the proposed NEI-exemplar inpainting technique. For consistency, we use similar notations as in [22].

Figure 4(c) illustrates the elemental image $\left(I^{0}\right)$ under consideration, while Fig. 4(a) and (b) are two left hand-side neighboring elemental images $\left(I^{1}\right.$ and $\left.I^{2}\right)$. The red colored region $\left(\Omega^{0}\right)$ in Fig. 4(c) represents the occluding object region which has to be inpainted by the proposed method, and $\partial \Omega^{0}$ denotes its boundary. The regions $I^{0}-\Omega^{0}, I^{1}-\Omega^{1}$ and $I^{2}-\Omega^{2}$ represent the source regions which provide for the samples used in the inpainting. The inpainting process starts from the boundary of $\Omega^{0}$, i.e., $\partial \Omega^{0}$. That is, for a point $p$ on $\partial \Omega^{0}$, we put a square patch $\psi_{p}$ centered at $p$. This square patch contains the region $\psi_{p} \cap\left(I^{0}-\Omega^{0}\right)$ for which the data is already filled, and the region $\psi_{p} \cap \Omega^{0}$ in which the data has to be filled by the NEI-exemplar inpainting. The region is filled from the patch lying in the source region which region is most similar to the already filled region $\psi_{p} \cap\left(I^{0}-\Omega^{0}\right)$. Here, we denote the center of the patch in the source region as $q$. The top row in Fig. 4 represents the first inpainting step, while the bottom row represents the second inpainting step. It is assumed in the top row that the most similar square patch $\left(\psi_{q}^{1}\right)$ is found in Fig. 4(b), while the patch $\psi_{q}^{2}$ in Fig. 4(a) could also be a candidate. The partial part in the patch $\psi_{q}^{1}$ which corresponds to the occluding region part in $\psi_{p}$ is copied into $\psi_{p} \cap \Omega^{0}$. By filling in the region $\psi_{p} \cap \Omega^{0}$, a partial filling of the whole occluding region is achieved as can be seen in Fig. 4(d). Likewise, the bottom row in Fig. 4 shows the second partial filling of the occluding region by the same procedure. Here, it is assumed that the most similar square patch is found in Fig. 4(e). The result of filling in the occluding region inside $\psi_{p}$ in Fig. 4(g) is shown in Fig. 4(h). The partial filling

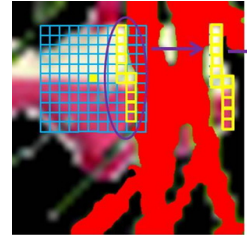

(a)

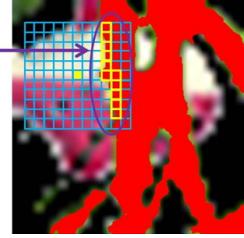

(b)

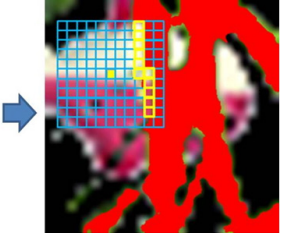

(c)
FIG. 5. Exemplary image explaining the procedure of filling in the missing region by the NEI-exemplar based inpainting method (a) neighboring elemental image (b) elemental image under consideration (c) elemental image after the first iteration of the filling-in procedure.

process is repeated until the occluding region becomes totally filled.

As can be seen in Fig. 4, unlike the original exemplar based inpainting technique, the best-match sample is found from the neighboring elemental images and not from the elemental image under consideration. In other words, the source region lies in neighboring elemental images. Therefore, the problem is formulated as

$$
\psi_{\hat{q}}^{k}=\arg \min _{\psi_{q}^{k} \in \Phi^{k}} d\left(\psi_{p}^{0} \cap\left(I^{0}-\Omega^{0}\right),\left\{\psi_{q}^{k} \cap\left(I^{k}-\Omega^{k}\right)\right\}_{(k=-n \sim n)}\right)
$$

where $k$ denotes the $k$-th neighboring elemental image. The function $\mathrm{d}\left(\Omega_{A}, \Omega_{B}\right)$ in (5) is a distance function that calculates the similarity distance between the two patch regions $\Omega_{A}$ and $\Omega_{B}$. As in [22], we let the distance function $d$ be the sum of squared differences (SSD) of the already filled pixels in the two patch regions.

Figure 5 is an exemplary image which shows how the proposed method fills in the data missing region. Figure 5 (b) is the elemental image under consideration, where the red region shows the data missing region. An $11 \times 11$ window is applied on Fig. 5(b), which searches for the window which has the most similar region in the neighboring elemental image (Fig. 5(a)). Then, the non-occluded region in the window in Fig. 5(a) is copied to the region which corresponds to the occluding region in Fig. 5(b). Figure 5(c) shows the copied result. Thus, a part of the occluding region has been recovered by the NEI-exemplar based inpainting. This procedure is iteratively applied to the elemental image in Fig. 5(b) until all the occluding regions are recovered.

After all the occluding regions are recovered, the CIIR is applied on the recovered elemental image array to result in the reconstructed 3-D image.

\section{EXPERIMENTS AND RESULTS}

The setup of the pickup stage in our experiments is as shown in the 'pickup stage' diagram shown in Fig. 1. The target 3-D objects are the 'faces' which are occluded by a 


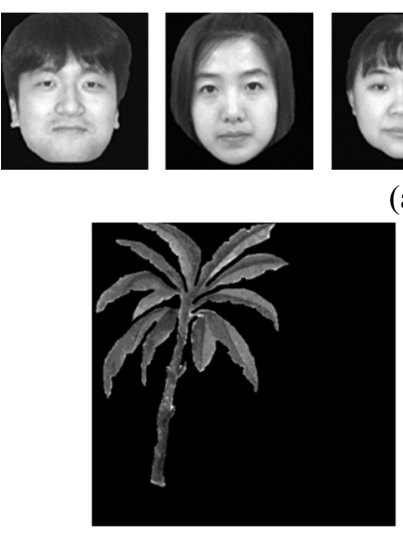

(b)

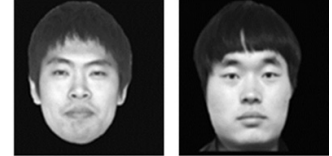

(a)

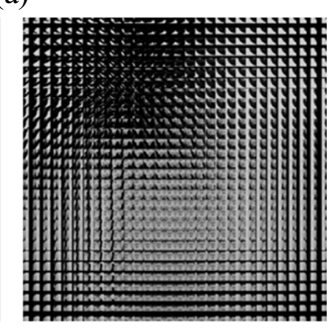

(c)
FIG. 6. Test images (a) five 'face' images used as the target object in the experiment (b) occluding object (c) elemental image array of the fifth 'face' image with occlusion.

'tree'. The target and the occluding objects are located $Z_{T}$ $=50 \mathrm{~mm}$ and $Z_{O}=30 \mathrm{~mm}$ from the lenslet array away, respectively. We used five different 'face' images as the target objects which are shown in Fig. 6(a). The occluding 'tree' object is shown in Fig. 6(b).

The lenslet array used in the experiments has $30 \times 30$ lenslets where each lenslet has a diameter of $5 \mathrm{~mm}$. Each lenslet has a resolution of $30 \times 30$ pixels, and therefore the total number of pixels in the lenslet array becomes $900 \times$ 900 pixels. Using this lenslet array, the partially occluded 'face' object was recorded to result in the $900 \times 900$ pixel elemental image array shown in Fig. 6(c).

First, the MC-DE method is applied to the recorded elemental images to obtain the depth field image. This is shown in Fig. 2 for the case of using the fifth face image in Fig 6(a). Then, the level set bimodal segmentation is applied to the depth map of Fig. 2 to obtain the mask map. Here, we let the parameter $\alpha$ in (2) be 1, so that the mask values in the mask map converge to 1 or -1 depending on whether the corresponding pixel belongs to the object region or background region. The final mask map is shown in Fig. 3(h).

Finally, we applied the proposed NEI-exemplar inpainting method using the mask map as the indication map of the data missing region. Figure 7 compares the inpainting result of the linear inpainting method and the proposed method. Figure 7(a) shows the elemental image array where the occluding object has been removed but the occluding region is left as a data missing region. Figure 7(b) and (c) show the elemental image arrays where the data missing region is filled in by the original linear inpainting and the proposed NEI-exemplar inpainting method, respectively. It can be seen that the linear inpainting method can fill in the missing data, but also results in some blurry artifacts. In comparison, the proposed NEI-exemplar inpainting method reconstructs the textural data in the missing region better than the linear inpainting method.

Next, we reconstructed the 3-D plane images using the CIIR method as shown in Fig. 8. Figure 8 shows the 3-D

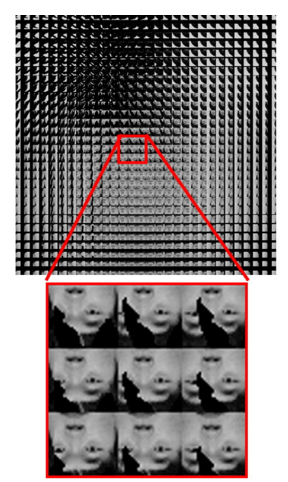

(a)

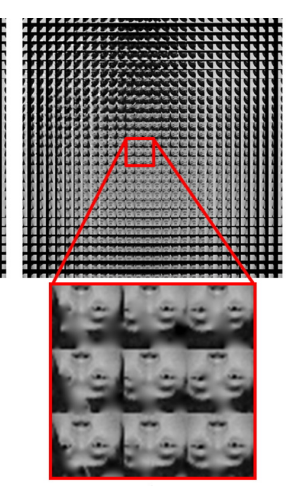

(b)

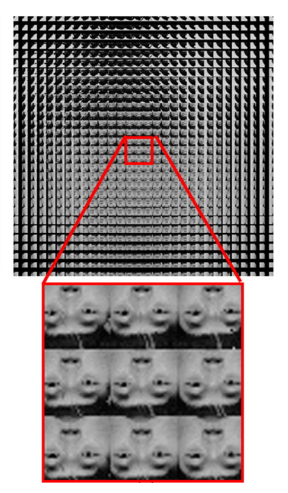

(c)
FIG. 7. Elemental images (a) before inpainting (b) after inpainting with original linear inpainting (c) after inpainting with proposed NEI-exemplar based inpainting.

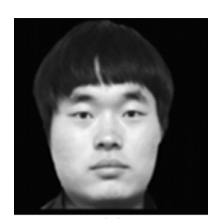

(a)

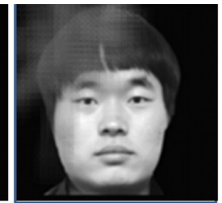

(b)

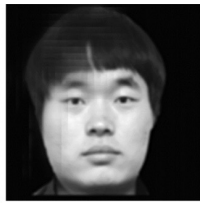

(c)

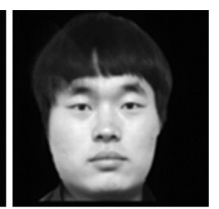

(d)
FIG. 8. (a) Original 3-D plane. Reconstructed 3-D plane images by (b) the conventional CIIR (c) the LI-CIIR method (d) the proposed method.

plane images of the fifth 'face' image reconstructed by different methods. It can be observed from Fig. 8(b), that the occlusion produces serious noises in the reconstructed image. The LI-CIIR can relieve the problem to a large extent as can be seen in Fig. 8(c). However, there still remain some errors in the brightness values in regions where the occluding object has occluded the target object. This is due to the blurring artifact in the elemental images caused by the linear inpainting. In comparison, the proposed method can improve the visual quality of the reconstructed image as can be seen in Fig. 8(d).

We measured the peak signal-to-noise ratio (PSNR) for all the reconstructed plane images for a quantitative comparison between the different methods. Figure 9 shows the PSNR results for the five face images used in the experiment. The linear inpainting based CIIR and the proposed NEI exemplar based inpainting using CIIR show high PSNR improvements over the conventional CIIR method. While both of the inpainting based CIIR methods show large improvements over the conventional CIIR, the proposed method shows a higher PSNR improvement than the linear inpainting based CIIR, thus revealing the superiority to the linear inpainting based CIIR.

Finally, we performed an experiment on an elemental image array which is obtained by a pickup setup as shown in Fig. 10. There are two target objects with different depths where 'target object 1 ' lies at $Z_{T 1}=50 \mathrm{~mm}$ and 'target object 2' at $Z_{T 2}=60 \mathrm{~mm}$. These targets are occluded by the 'tree' object which lies at $Z_{O}=30 \mathrm{~mm}$. Furthermore, the 


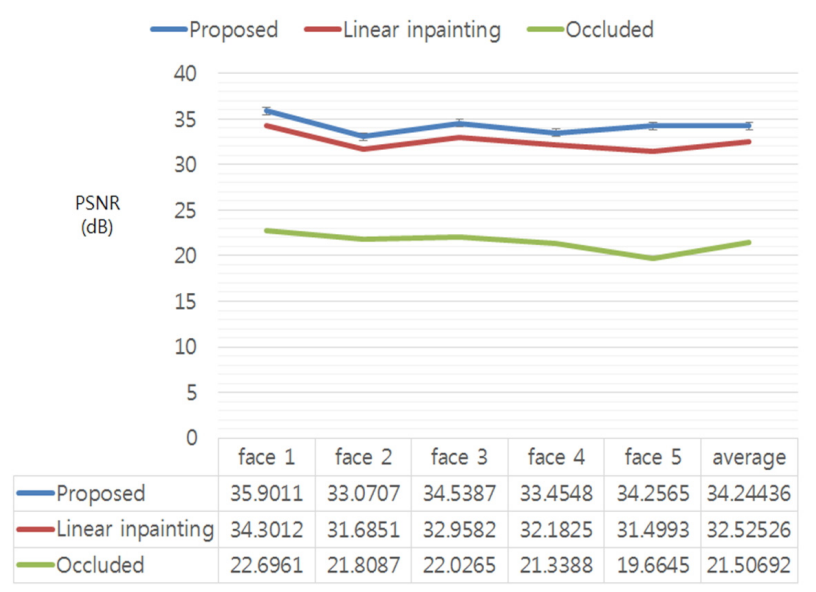

FIG. 9. Comparing the PSNR values of the reconstructed 3-D images. 'target object 2' has a textured surface.

Again, we removed the occluding 'tree' object from the elemental image array and reconstructed the 3-D image at planes with depths of $z=50 \mathrm{~mm}$ and $z=60 \mathrm{~mm}$, respectively. The second and the third rows of Fig. 11 show the reconstructed 3-D images reconstructed at $z=50 \mathrm{~mm}$ and $z=60 \mathrm{~mm}$, respectively. Only the target object which has the same depth as the plane at which the 3-D image is reconstructed becomes focused while the target object having a different depth becomes out of focus. The third row has some blurred region in it, which is due to the fact that 'target object 1' lies before 'target object 2', and because we only eliminated the occluding object and not 'target object 1'. Here, it can be seen that the difference in the PSNR value between the proposed method and the linear inpainting based CIIR is larger than shown in Fig. 9.

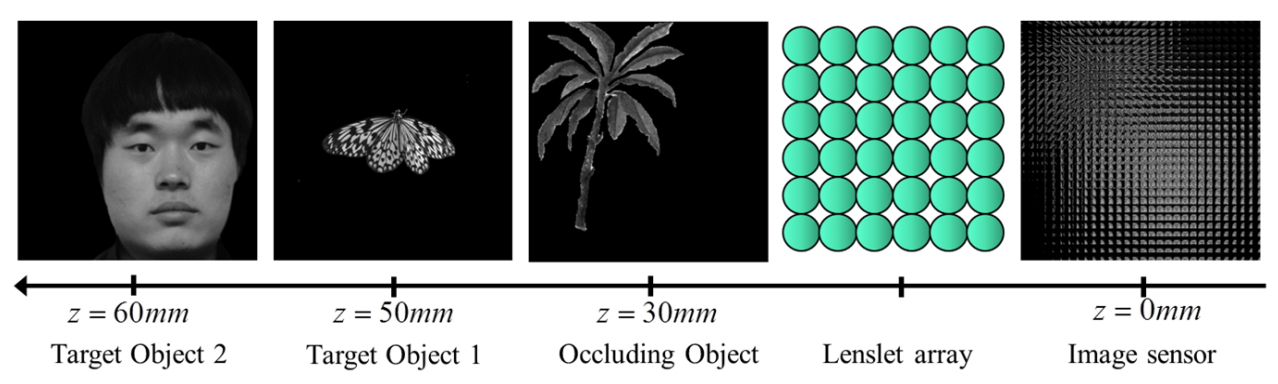

FIG. 10. Pickup process of two target objects with different depths and one occluding object.
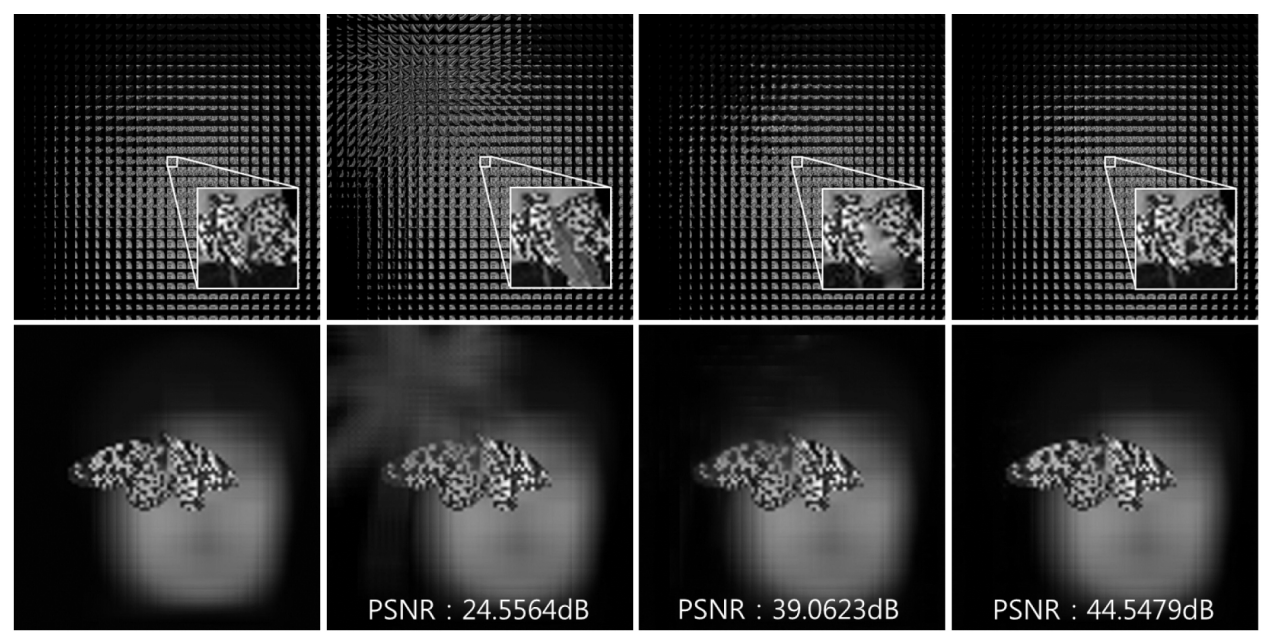

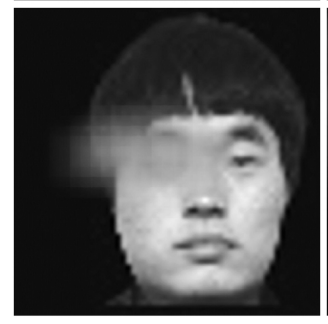

(a)

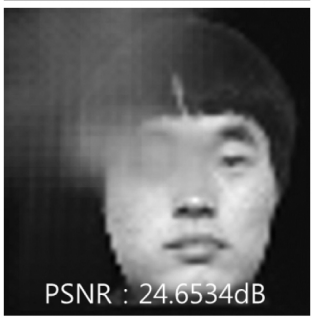

(b)

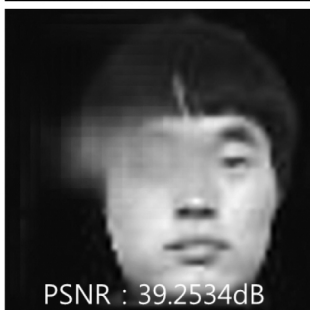

(c)

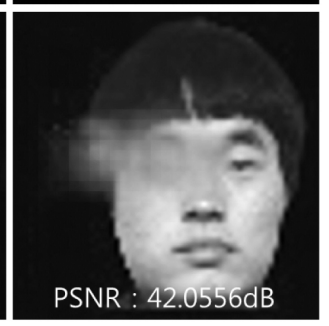

(d)

FIG. 11. First row: Elemental image array. Second row: 3-D plane reconstructed at $z=50 \mathrm{~mm}$ Third row: 3-D plane reconstructed at $z=60 \mathrm{~mm}$ (a) without occlusion (b) with conventional CIIR (c) with LI-CIIR method (d) with proposed method. 
This is due to the fact that 'target object 1' has a textured surface which the proposed method can recover much better than the linear inpainting based CIIR.

\section{CONCLUSION}

We proposed a method which can recover not only smooth data but also textured data in the data missing region caused by the partial occlusion of the occluding object. To this aim, the proposed method fills in the data missing region with exemplars obtained from neighboring elemental images. It has been shown experimentally, that the proposed method can recover textural regions in the elemental images, while other conventional CIIR methods including the linear inpainting based CIIR method cannot. This results in a 3-D image reconstruction better than those using conventional methods. It is expected that the proposed method can be used in several applications where the target object has to be clearly visualized and recognized in spite of a partial occlusion.

\section{ACKNOWLEDGMENT}

This work was supported by the Basic Science Research Program (2013R1A1A4A01007868) through the National Research Foundation of Korea (NRF) funded by the Ministry of Education, Science and Technology.

\section{REFERENCES}

1. A. Stern and B. Javidi, "Three-dimensional image sensing, visualization, and processing using integral imaging," Proc. IEEE 94, 591-607 (2006).

2. S.-H. Hong, J.-S. Jang, and B. Javidi, "Three-dimensional volumetric object reconstruction using computational integral imaging," Opt. Express 12, 483-491 (2004).

3. J.-H. Park, K. Hong, and B. Lee, "Recent progress in threedimensional information processing based on integral imaging," Appl. Opt. 48, H77-H94 (2009).

4. D.-H. Shin, E.-S. Kim, and B. Lee, "Computational reconstruction technique of three-dimensional object in integral imaging using a lenslet array,” Jpn. J. Appl. Phys. 44, 80168018 (2005).

5. D.-H. Shin, M.-W. Kim, H. Yoo, J.-J. Lee, B. Lee, and E.-S. Kim, "Improved viewing quality of 3-D images in computational integral imaging reconstruction based on round mapping model," ETRI J. 29, 649-654 (2007).

6. H. Yoo, "Artifact analysis and image enhancement in three-dimensional computational integral imaging using smooth windowing technique," Opt. Lett. 36, $2107-2109$ (2011).

7. D.-H. Shin and E.-S. Kim, "Computational integral imaging reconstruction of 3D object using a depth conversion technique," J. Opt. Soc. Korea 12, 131-135 (2008).

8. J.-Y. Jang, D. Shin, and E.-S. Kim, "Optical three-dimensional refocusing from elemental images based on a sifting property of the periodic $\delta$-function array in integral-imaging," Opt. Express 22, 1533-1550 (2014).

9. J.-Y. Jang, J.-I. Ser, S. Cha, and S.-H. Shin, "Depth extraction by using the correlation of the periodic function with an elemental image in integral imaging," Appl. Opt. 51, 3279-3286 (2012).

10. D.-H. Shin and H. Yoo, "Scale-variant magnification for computational integral imaging and its application to 3D object correlator," Opt. Express 16, 8855-8867 (2008).

11. C. Kim, S.-C. Park, and E.-S. Kim, "Computational integralimaging reconstruction-based 3-D volumetric target object recognition by using a 3-D reference object," Appl. Opt. 48, H95-H104 (2009).

12. D.-C. Hwang, D.-H. Shin, S.-C. Kim, and E.-S. Kim, "Depth extraction of three-dimensional objects in space by the computational integral imaging reconstruction technique," Appl. Opt. 47, D128-D135 (2008).

13. M. Cho and B. Javidi, "Three-dimensional visualization of objects in turbid water using integral imaging," J. Display Technol. 6, 544-547 (2010).

14. J.-Y. Jang, S.-P. Hong, D. Shin, B.-G. Lee, and E.-S. Kim, "3D image correlator using computational integral imaging reconstruction based on modified convolution property of periodic functions," J. Opt. Soc. Korea 18, 388-394 (2014).

15. M. Zhang, Y. Piao, and E.-S. Kim, "Occlusion-removed scheme using depth-reversed method in computational integral imaging," Appl. Opt. 49, 2571-2580 (2010).

16. D.-H. Shin, B.-G. Lee, and J.-J. Lee, "Occlusion removal method of partially occluded 3D object using sub-image block matching in computational integral imaging," Opt. Express 16, 16294-16304 (2008).

17. B.-G. Lee, Liliana, and D. Shin, "Enhanced computational integral imaging system for partially occluded 3D objects using occlusion removal technique and recursive PCA reconstruction," Opt. Commun. 283, 2084-2091 (2010).

18. J.-J. Lee, B.-G. Lee, and H. Yoo, "Image quality enhancement of computational integral imaging reconstruction for partially occluded objects using binary weighting mask on occlusion areas," Appl. Opt. 50, 1889-1893 (2011).

19. J.-J. Lee, D. Shin, and H. Yoo, "Image quality improvement in computational reconstruction of partially occluded objects using two computational integral imaging," Opt. Commun. 304, 96-101 (2013).

20. B.-G. Lee, B.-S. Ko, S.-H. Lee, and D. Shin, "Computational integral imaging reconstruction of a partially occluded threedimensional object using an image inpainting technique," J. Opt. Soc. Korea 19, 248-254 (2015).

21. D. Garcia, "Robust smoothing of gridded data in one and higher dimensions with missing values," Comput. Stat. Data An. 54, 1167-1178 (2010).

22. A. Criminisi, P. Pérez, and K. Toyama, "Object removal by exemplar-based image inpainting," IEEE Trans. Image Process. 13, 1200-1212 (2004).

23. T.-K. Ryu, B.-G. Lee, and S.-H. Lee, "Mutual constraint using partial occlusion artifact removal for computational integral imaging reconstruction," Appl. Opt. 54, 4147-4153 (2015).

24. S.-H. Lee and J.-K. Seo, "Level set-based bimodal segmentation with stationary global minimum," IEEE Trans. Image Process. 15, 2843-2852 (2006). 